02

\title{
Влияние электрон-фононного взаимодействия на фотолюминесценцию оксида титана в ближней инфракрасной области
}

\author{
(C) С.В. Булярский ${ }^{1}$, В.С. Горелик ${ }^{2}$, Г.Г. Гусаров ${ }^{1}$, Д.А. Коива ${ }^{1}$, А.В. Лакалин ${ }^{1}$ \\ ${ }^{1}$ Институт нанотехнологий микроэлектроники РАН, \\ 119991 Москва, Россия \\ 2 Физический институт им. П.Н. Лебедева РАН, \\ 119991 Москва, Россия \\ e-mail: bulyar2954@mail.ru
}

Поступила в редакцию 29.07.2019 г.

В окончательной редакции 06.02.2020 г.

Принята к публикации 07.02.2020 г.

\begin{abstract}
Исследован рекомбинационный центр $\mathrm{Ti}^{+3}$ - вакансия кислорода, который играет важную роль в процессах фотокатализа. При возбуждении оксида титана лазером с длиной волны $785 \mathrm{~nm}$ наблюдалась полоса фотолюминесценции с максимумом $1.236 \mathrm{eV}$. Данная полоса появляется после кристаллизации аморфной пленки диоксида титана в вакууме при температуре $650^{\circ} \mathrm{C}$ в течение $30 \mathrm{~min}$. Форма полосы исследована методом моментов и определены параметры ее электрон-фононного взаимодействия: энергия чисто электронного перехода $-1.28 \mathrm{eV}$, фактор Хуанга и Риса -2 , величина тепловыделения $0.04 \mathrm{eV}$. Эти значения можно использовать для вычисления параметров кинетических коэффициентов электронных переходов в сильных электрических полях.
\end{abstract}

Ключевые слова: диоксид титана, фотолюминесценция, вакансия кислорода, электрон-фононное взаимодействие.

DOI: $10.21883 /$ OS.2020.05.49315.239-19

\section{Введение}

Диоксид титана $\left(\mathrm{TiO}_{2}\right)$ является технологически важным оксидом переходного металла, поскольку обладает рядом уникальных свойств, в частности: широкая запрещенная зона [1], высокий показатель преломления [2,3], большая диэлектрическая проницаемость $[4,5]$. Он также представляет интерес с точки зрения фотохимических свойств, что позволяет его использовать для разложения воды на водород и кислород [6]. Кроме того, на сегодняшний день активно исследуются вопросы деградации органических молекул. В этой области $\mathrm{TiO}_{2}$ применяется в качестве материала для очистки сточных вод [7], дезинфекции [8], самоочищающегося покрытия [9], антикоррозионной защиты [10]. Диоксид титана также используется в солнечных элементах [11].

Оксид титана может быть использован также в качестве датчика контроля воздушно-топливной смеси в автомобильных двигателях [12]. Высокая диэлектрическая проницаемость расширяет возможности применения $\mathrm{TiO}_{2}$ в электронике, например, в качестве конденсатора и запоминающего устройства. В нашей повседневной жизни диоксид титана используется практически в каждом красителе из-за его высокого показателя преломления. Чистый $\mathrm{TiO}_{2}$ нетоксичен, легко диспергируется, и может быть использован как пищевая добавка [13], в косметических продуктах, а также в фармацевтике [14].
Дефекты и поверхностные состояния $\mathrm{TiO}_{2}$ оказывают большое влияние на его электрические, химические, а также оптические свойства. Содержание и состав дефектов зависят от технологий подготовки материала. Поэтому необходимо выявить методы контроля результатов технологических процессов и научится проектировать содержание и состав дефектов в $\mathrm{TiO}_{2}$.

Диоксид титана обладает широкой областью гомогенности, поэтому важную роль играют дефекты, связанные с вакансией кислорода. Одним из таких дефектов является рекомбинационный центр $\mathrm{Ti}^{+3}-$ вакансия кислорода [15], который играет важную роль в фотокатализе. Данный центр имеет известные полосы люминесценции. Поэтому для контроля этих точечных дефектов предлагается проводить исследование фотолюминесцентных свойств материала. Измерение спектров люминесценции наряду со спектрами диффузного отражения является чувствительным методом анализа дефектности тонких пленок и их стабильности к действию квантов и заряженных частиц. Поэтому исследования спектров для пленок $\mathrm{TiO}_{2}$ в исходном состоянии сразу после синтеза и после технологических обработок могут способствовать пониманию закономерностей дефектообразования в данном материале и определению путей получения материала с нужными свойствами. При этом важно получать информацию не только о спектральном положении пика, но и о его форме. Для этого необходимо вычислять моменты полосы излучения и параметры электрон-фо- 
нонного взаимодействия, которые позволяют оценить вероятности поглощения и излучения, в том числе в сильных электрических полях $[16,17]$.

Исследований спектров фотолюминесценции диоксида титана в ближней ИК области чрезвычайно мало. Работы, посвященные влиянию излучений на такие спектры, практически отсутствуют, параметры электронфононного взаимодействия для них не исследовались до настоящего времени. Цель настоящей работы определение параметров люминесцентных свойств в ближней ИК области спектра тонкопленочных образцов кристаллического диоксида титана с учетом электронфононного взаимодействия.

\section{Результаты экспериментов}

Формирование пленок осуществлялось посредством осаждения оксида титана на термически окисленные пластины монокристаллического кремния $n$-типа $\left(\rho_{n} \sim 0.001-0.005 \Omega \cdot \mathrm{cm}\right)$ толщиной $460 \mu \mathrm{m}$. Толщина окисла $-1.7 \mu \mathrm{m}$. Вся серия подложек на этапе подготовки подвергалась гидромеханической очистке от загрязнений на установке DNS Screen.

Для осаждения тонких пленок использовалось высокочастотное магнетронное распыление, благодаря чему удалось получить пленки заданного состава, в том числе стехиометрического. Расстояние между мишенью и подложкой для всех экспериментов было зафиксировано на уровне $45 \mathrm{~mm}$. Напыление тонкой пленки $\mathrm{TiO}_{2}$ осуществлялось из мишени металлического титана чистотой 99.97\% в смеси аргона с кислородом в определенной пропорции. Непосредственно перед напылением пленок на подложку мишень предварительно распыляли на специальную заслонку, чтобы удалить с нее слой окисла, образовавшийся во время предыдущих процессов.

Остаточное давление в вакуумной камере составляло $5 \cdot 10^{-6} \mathrm{~mm} \mathrm{Hg}$. Рабочее давление фиксировалось на уровне $2 \cdot 10^{-4} \mathrm{~mm} \mathrm{Hg}$ при распылении в среде аргон/кислород. Подача рабочих газов в камеру осуществлялась при помощи двух автоматических регуляторов расхода газа с точностью задания и точностью поддержания газовой смеси $\pm 0.8 \%$, что позволяло задавать концентрацию газов в рабочей газовой смеси аргонкислород с точностью $\pm 1.6 \%$. Скорости подачи газов составляли $20 \mathrm{~cm}^{3} / \mathrm{min}$ для $\mathrm{Ar}$ и $5 \mathrm{~cm}^{3} / \mathrm{min}$ для $\mathrm{O}_{2}$. Процесс проводился при $200 \mathrm{~W}$ мощности магнетрона.

Толщина формируемых тонкопленочных структур может регулироваться либо заданием значения скорости распыления и контролироваться по кварцевому датчику, либо длительностью процесса распыления. Толщина полученных структур составляла приблизительно $100 \mathrm{~nm}$.

После осаждения часть образцов была подвергнута отжигу в вакуумной камере при температуре $650^{\circ} \mathrm{C}$ в течение $30 \mathrm{~min}$.

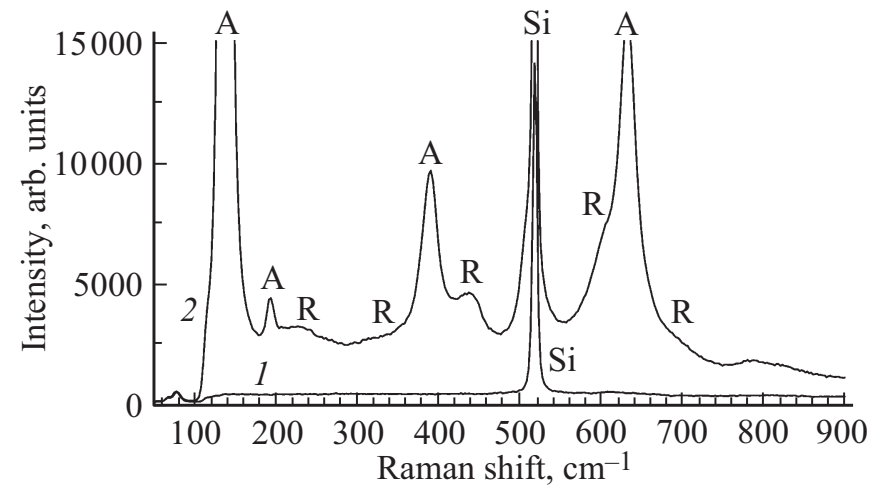

Рис. 1. Спектры комбинационного рассеяния света диоксида титана. Возбуждение лазером с длиной волны $405 \mathrm{~nm} .1-$ образец сразу после магнетронного напыления; 2 - после отжига при температуре $650{ }^{\circ} \mathrm{C}$ в течение $30 \mathrm{~min}$; A - пики, относящиеся к анатазу; R - пики, относящиеся к рутилу, $\mathrm{Si}-$ пики, относящиеся к кремниевой подложке.

Толщины пленок были определены с помощью профилометра, а их элементный состав - методом оже-электронной спектроскопии. Спектры фотолюминесценции регистрировались при комнатной температуре с помощью рамановского спектрометра. В качестве источника возбуждения использовался лазер с длиной волны излучения $785 \mathrm{~nm}$.

Исследование спектров комбинационного рассеяния света показало, что после отжига в вакууме при температуре $650^{\circ} \mathrm{C}$ в течение $30 \mathrm{~min}$ диоксид титана переходит в кристаллическое состояние преимущественно в аллотропном состоянии анатаза, рис. 1 (положения пиков идентифицировались в соответствии с работой [18]). Сразу после магнетронного напыления пленка диоксида титана имеет аморфную структуру. Комбинационное рассеяние света от нее отсутствует, а в спектре проявляются только линии кремниевой подложки. После отжига происходит кристаллизация образца преимущественно в аллотропной модификации анатаза.

\section{Спектр фотолюминесценции диоксида титана в ближней ИК области}

Спектр фотолюминесценции исследуемого образца, отожженного при температуре $650^{\circ} \mathrm{C}$, представлен на рис. 2. Полоса $1.09-1.42 \mathrm{eV}$ с максимумом вблизи $1.236 \mathrm{eV}(\lambda=1003 \mathrm{~nm})$ обусловлена люминесценцией центра. Понять природу наблюдаемой полосы излучения можно, если привлечь диаграмму электронных переходов, предложенную в работе [15] для дефекта, который возникает в результате взаимодействия иона $\mathrm{Ti}^{+3}$ и вакансии кислорода.

Для анализа электронно-колебательных переходов в образцах диоксида титана был использован метод моментов [19]. Этот метод применим для расчета контуров спектральных полос примесного центра в кристалле, а 


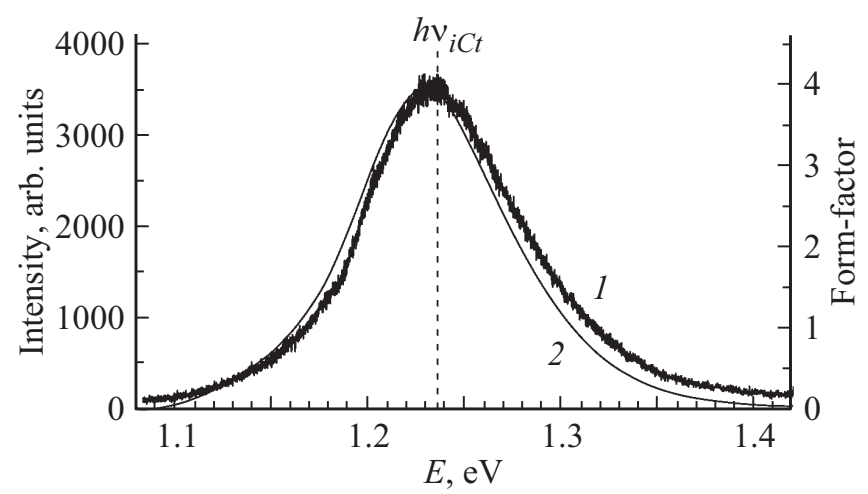

Рис. 2. Спектр фотолюминесценции отожженного диоксида титана (1) (длина волны возбуждающего лазера $785 \mathrm{~nm}$ ) и его форм-фактор (2), рассчитанный по формуле (2).

также сложной молекулы. Дальнейшее развитие метод моментов получил в работах $[20,21]$, в которых был применен к расчету различных моделей центров кристаллофосфоров. В работах $[16,17,22,23]$ данный метод был модифицирован и применялся для анализа спектров люминесценции и вычисления вероятности излучательных и безызлучательных переходов в полупроводниковых материалах $\left(\mathrm{GaAs}, \mathrm{CdI}_{2} \mathrm{~S}_{4}\right)$.

Простейшей моделью, описывающей распределение интенсивностей в сплошных спектральных полосах, возникающих при электронно-колебательных переходах, является однокоординатная модель [16,20-23]. В основе однокоординатной модели лежит адиабатическое приближение и метод нормальных координат. Эта модель учитывает взаимодействие только с одним нормальным колебанием (т.е. с фононом одного типа). Потенциальная конфигурационно-координатная диаграмма, построенная в форме, предложенной в работах $[19,20]$, представлена на рис. 3. В соответствии с работами $[22,23]$ верхняя $U_{u}$ и нижняя $U_{g}$ потенциальные кривые обозначают адиабатические потенциалы. По оси ординат откладывается энергия колебательного центра, состоящая из энергии электрона и упругой энергии. Адиабатический потенциал $U_{g}$ соответствует основному состоянию центра, образованного ионом $\mathrm{Ti}^{+3}$ и вакансией кислорода, $U_{u}-$ возбужденному. $E_{0 C}-$ энергия чисто электронного перехода, равная минимальному энергетическому промежутку для перехода из основного состояния в возбужденное.

Возбуждение электрона с центра рекомбинации в зону проводимости осуществлялось лазером с длиной волны излучения $785 \mathrm{~nm}$. Энергия кванта излучения данного лазера, $1.58 \mathrm{eV}$, меньше половины ширины запрещенной зоны $\mathrm{TiO}_{2}$, и поэтому возможен только один переход с уровня $E_{0}$ в зону проводимости $E_{C}$. Этот переход является мгновенным и вертикальным в соответствии с принципом Франка-Кондона. Он переводит электронноколебательную систему, которая изображается конфигурационной диаграммой на рис. $3, a$, в возбужденное со- стояние. В течение некоторого времени система релаксирует в состояние квазиравновесия, которое изображается точкой А. Из этой точки возможен излучательный переход в точку В, после чего система релаксирует в состояние равновесия (в точку С). С учетом электронфононного взаимодействия чисто электронный переход равен энергии излучательного перехода, увеличенного на величину стоксовых потерь $S \hbar \omega_{g}$, либо энергии поглощения, уменьшенной на величину стоксовых потерь $S \hbar \omega_{u}$. Поэтому:

$$
\begin{gathered}
h v_{i C_{0}}=E_{0 C}-S \hbar \omega_{g}, h v_{a C 0}=E_{0 C}+S \hbar \omega_{u}, \\
h v_{a C 0}-h v_{i C 0}=\left(S \hbar \omega_{g}+S \hbar \omega_{u}\right),
\end{gathered}
$$

где $h v_{a c 0}-$ энергия максимума поглощения при переходе из основного состояния ,g“ в возбужденное „и“, $h v_{i c 0}-$ энергия максимума излучения при переходе из возбужденного состояния в основное.

Рекомбинационный центр может обмениваться носителями заряда с валентной зоной, как это показано на рис. $3, b$. Так как энергия поглощения и излучения отличаются на величину тепловыделения, то это учтено
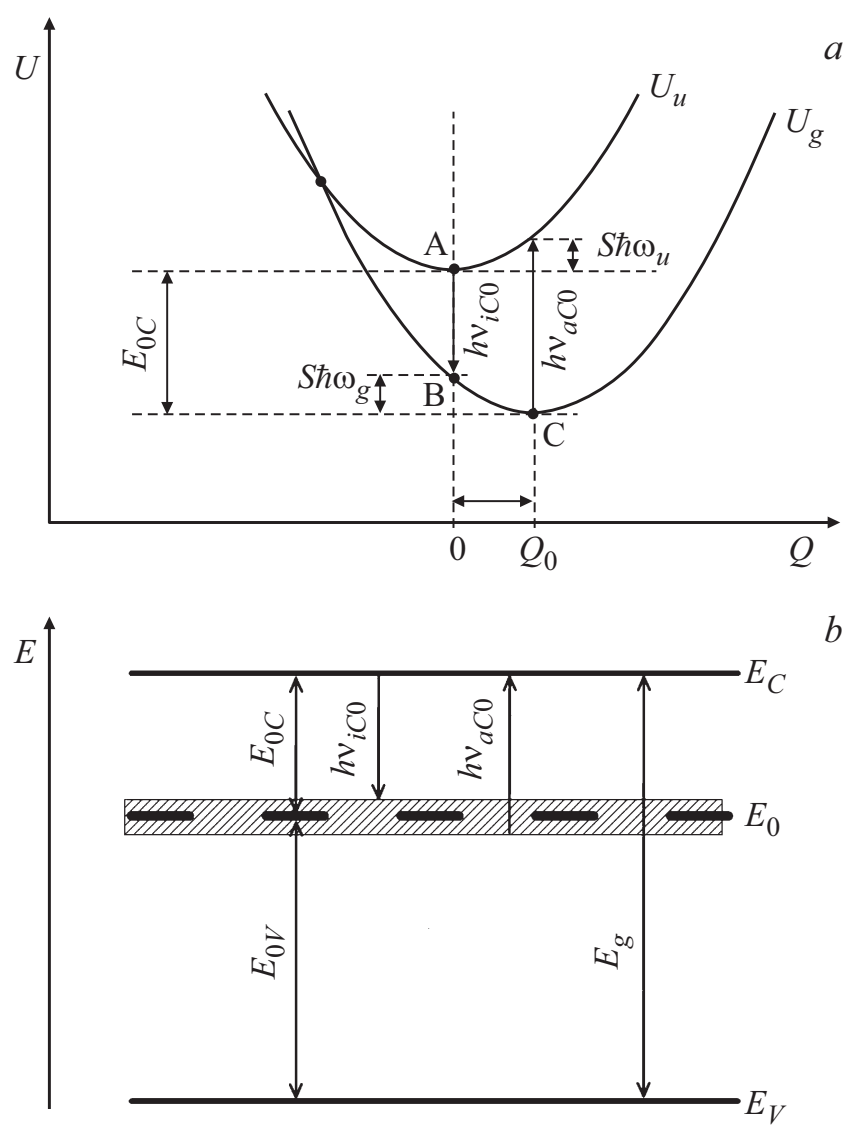

Рис. 3. Потенциальная конфигурационно-координатная диаграмма однокоординатной модели $(a)$ и зонная диаграмма $(b)$. $g$ - основное (невозбужденное) состояние, $u$ - возбужденное состояние; $Q-$ конфигурационная координата, $Q_{0}-$ координата минимума потенциальной энергии основного состояния; $U_{g}(Q)$ и $U_{u}(Q)-$ потенциальные энергии основного и возбужденного состояний соответственно. 
на рис. $3, b$ тем, что энергетическое положение рекомбинационного центра показывается не линией, а полосой, ширина которой равна тепловыделению. Энергия чисто электронных переходов расположена внутри этой полосы. Энергия перехода $E_{0 V}$ известна и составляет величину около $2 \mathrm{eV}[15]$. Энергия перехода $h v_{i c 0}$ наблюдалась нами экспериментально и равна $1.236 \mathrm{eV}$ (рис. 2). Сумма энергий этих двух переходов приблизительно равна ширине запрещенной зоны $\mathrm{TiO}_{2}$ в модификации анатаза $3.23 \mathrm{eV}$ [24]. Заметим, что ширина запрещенной зоны диоксида титана зависит от технологии получения и обработки его пленки.

Несмотря на свою приближенность, однокоординатная модель объясняет: наличие стоксовых потерь (когда длина волны максимума излучения больше, чем поглощения); уширение полос оптического поглощения и излучения; температурный сдвиг и уширение линий; существование многофононных безызлучательных переходов.

В работе [20] в приближении Франка-Кондона получены выражения для первых моментов полос поглощения и излучения. Для фотолюминесценции моменты имеют вид

$$
\begin{gathered}
M_{0}=1 \\
M_{1}=\left[E_{0}+S \hbar \omega_{g}=\frac{k \tau_{u}}{2}\left(\frac{\omega_{g}^{2}}{\omega_{u}^{2}}-1\right)\right] \\
\left\langle M_{2}\right\rangle=\frac{k \tau_{u}}{2}\left(\frac{4 S \hbar \omega_{g}^{3}}{\omega_{u}^{2}}+k \tau_{u}\left(\frac{\omega_{g}^{2}}{\omega_{u}^{2}}-1\right)^{2}\right), \\
\left\langle M_{3}\right\rangle=\left[\frac{\hbar^{2}}{2}\left(2 S \hbar \omega_{g}^{3}+k \tau_{u} \omega_{u}^{2}\left(\frac{\omega_{g}^{2}}{\omega_{u}^{2}}-1\right)^{2}\right)\right. \\
\left.+\frac{3}{2}\left(k \tau_{u}\right)^{2} \frac{4 S \hbar \omega_{g}^{3}}{\omega_{u}^{2}}\left(\frac{\omega_{g}^{2}}{\omega_{u}^{2}}-1\right)+\left(k \tau_{u}\left(\frac{\omega_{g}^{2}}{\omega_{u}^{2}}-1\right)\right)^{3}\right] \\
k \tau_{u}=\frac{1}{2} \hbar \omega_{u} \operatorname{coth}\left(\frac{\hbar \omega_{u}}{2 k T}\right) .
\end{gathered}
$$

Здесь $M_{1}$ - начальный и $\left\langle M_{2}\right\rangle,\left\langle M_{3}\right\rangle$ - центральные моменты соответствующих порядков форм-фактора полосы излучения [22,23], $S$ - фактор Хуанга и Риса, имеющий смысл количества фононов, возникающих (исчезающих) в одном акте излучения (поглощения). Согласно работам [22,23], форм-фактор спектра излучения электронного перехода вычисляется по формуле

$$
f(\varepsilon)=\frac{I(\varepsilon)}{Z_{0} \varepsilon^{4}}
$$

Здесь $I(\varepsilon)$ - интенсивность люминесценции, $\varepsilon$ - энергия фотона, $Z_{0}-$ нулевой момент полосы излучения: $Z_{0}=\int I(\varepsilon) d \varepsilon$. Тогда начальные моменты форм-фактора равны:

$$
M_{n}=\int \varepsilon^{n} f(\varepsilon) d \varepsilon
$$

а центральные находятся по формулам [25]

$$
\left\langle M_{n}\right\rangle=\sum_{i=0}^{n}(-1)^{i}\left(\begin{array}{c}
n \\
i
\end{array}\right)\left(\frac{M_{1}}{M_{0}}\right)^{i} M_{n-i} .
$$

Уравнения (1) составлены для колебательных систем с различными значениями энергий переходов в основном $\left(E_{g r}=\hbar \omega_{g}\right)$ и возбужденном $\left(E_{u p}=\hbar \omega_{u}\right)$ состояниях. Численное решение (1) сводится к задаче многомерной оптимизации с целевой функцией:

$$
\begin{aligned}
& \Phi\left(S, E_{g r}, E_{u p}, E_{0}\right)=\left(\left\langle M_{2}\right\rangle-\left(\frac{1}{4}\right) E_{u p} \operatorname{coth}\left(\frac{E_{u p}}{2 E_{T}}\right)\right. \\
& \left.\times\left(4 \frac{S E_{g r}^{3}}{E_{u p}^{2}}+\left(\frac{1}{2}\right) E_{u p} \operatorname{coth}\left(\frac{E_{u p}}{2 E_{T}}\right)\left(\frac{E_{g r}^{2}}{E_{u p}^{2}}-1\right)^{2}\right)\right)^{2} \\
& +\left(M_{1}-S E_{g r}-E_{0}+\frac{1}{4} E_{u p} \operatorname{coth}\left(\frac{E_{u p}}{2 E_{T}}\right)\left(\frac{E_{g r}^{2}}{E_{u p}^{2}}-1\right)^{2}\right. \\
& +\left(\left\langle M_{3}\right\rangle-S E_{g r}^{3}-\left(\frac{1}{4}\right) E_{u p}^{3} \operatorname{coth}\left(\frac{E_{u p}}{2 E_{T}}\right)\left(\frac{E_{g r}^{2}}{E_{u p}^{2}}-1\right)^{2}\right. \\
& -\left(\frac{3}{2}\right) \operatorname{coth}\left(\frac{E_{u p}}{2 E_{T}}\right)^{2} S E_{g r}^{3}\left(\frac{E_{g r}^{2}}{E_{u p}^{2}}-1\right)^{2} \\
& \left.-\left(\frac{1}{8}\right) E_{u p}^{3} \operatorname{coth}\left(\frac{E_{u p}}{2 E_{T}}\right)^{3}\left(\frac{E_{g r}^{2}}{E_{u p}^{2}}-1\right)^{3}\right)^{2} \\
& +\left(E_{\max }-S E_{g r}-E_{0}+\left(\frac{1}{2}\right)_{E_{u p}}\right. \\
& \left.\times \operatorname{coth}\left(\frac{E_{u p}}{2 E_{T}}\right)\left(\frac{E_{g r}^{2}}{E_{u p}^{2}}-1\right)^{2}+\frac{E_{u p}}{2 \operatorname{coth}\left(\frac{E_{u p}}{2 E_{T}}\right)}\right)^{2}
\end{aligned}
$$

где $E_{T}=k T, E_{0}=\min _{x} U_{u}(x)-\min _{x} U_{g}(x), E_{\max }-$ максимум форм-фактора.

Для решения оптимизационной задачи был использован метод сопряженных направлений Пауэлла [26,27]. Особенностью численных алгоритмов многомерной оптимизации является появление множества наборов возможных решений, из которых необходимо выбрать подходящие, руководствуясь дополнительными критериями. В нашем случае в качестве дополнительных ограничений на возможные значения неизвестных были наложены требования положительности всех искомых значений, а также целочисленности параметра $S$. Кроме того, сузить область поиска позволили разумные ограничения, следующие из физического смысла задачи: $1.10 \mathrm{eV}<E_{0}<130 \mathrm{eV}, S \hbar \omega_{g} \leq 0.1 \mathrm{eV}, E_{g r} / E_{u p} \sim 1$. В качестве стартовой точки поиска было использовано 
решение упрощенной системы $\left(E_{g r}=E_{u p}=E\right)$ :

$$
\begin{gathered}
\left\langle M_{2}\right\rangle-E^{2} S \operatorname{coth}\left(\frac{E}{2 E_{T}}\right)=0, E^{3} S-\left\langle M_{3}\right\rangle=0, \\
M_{1}-E S-E_{0}=0 .
\end{gathered}
$$

Для пика $1.236 \mathrm{eV}(\lambda=1003 \mathrm{~nm})$ в спектре фотолюминесценции был рассчитан форм-фактор (рис. 2), для которого были определены моменты $M_{1}=1.233 \mathrm{eV}$, $\left\langle M_{2}\right\rangle=\sigma^{2}=2.24 \cdot 10^{-3}(\mathrm{eV})^{2}, \quad\left\langle M_{3}\right\rangle=1.63 \cdot 10^{-5}(\mathrm{eV})^{3}$ и коэффициент асимметрии $\gamma=\frac{\left\langle M_{3}\right\rangle}{\left\langle M_{2}\right\rangle^{\frac{3}{2}}}=0.153$. Используя найденные значения, затем была решена система (5) и получены значения $E=\hbar \omega=2.01 \cdot 10^{-2} \mathrm{eV}$, $E_{0}=1.19 \mathrm{eV}, \quad S=2$. Тогда величина тепловыделения (стоксовых потерь) $2 S \hbar \omega=4.02 \cdot 10^{-2} \mathrm{eV} \approx 0.040 \mathrm{eV}$.

Для исходной задачи (4) $\left(E_{g r} \neq E_{u p}\right)$ и формфактора были получены значения: $E_{g r}=2.04 \cdot 10^{-2} \mathrm{eV}$ и $E_{\text {up }}=2.01 \cdot 10^{-2}, \quad E_{0}=1.19 \mathrm{eV}, S=2$ и соответственно величина тепловыделения $\left(S \hbar \omega_{g}+S \hbar \omega_{u}\right)=$ $=4.05 \cdot 10^{-2} \mathrm{eV} \approx 0.041 \mathrm{eV}$. О присутствии в системе электрон-фононного взаимодействия можно судить по наличию легкой асимметрии графика форм-фактора по сравнению с функцией Гаусса, которая характеризуется ненулевым третьим моментом.

\section{Заключение}

Таким образом, в работе показано, что при отжиге в вакуумной камере при температуре $650^{\circ} \mathrm{C}$ в течение $30 \mathrm{~min}$ аморфная пленка диоксида титана кристаллизуется преимущественно в форме анатаза и появляется полоса люминесценции с максимумом $1.236 \mathrm{eV}$. Для наблюдения этой полосы $\mathrm{TiO}_{2}$ необходимо возбуждать лазером с длиной волны $785 \mathrm{~nm}$. В этом случае происходит возбуждение центра $\mathrm{Ti}^{+3}$ - вакансия кислорода [15]. Переходы с центра в валентную зону не возбуждаются, так как энергия квантов используемого лазера недостаточна. Последний переход достаточно хорошо описан в научной литературе [15] и создает полосу излучения с максимумом в красной области спектра и энергией максимума порядка $2 \mathrm{eV}$. В сумме энергия этих двух переходов близка к ширине запрещенной зоны анатаза. В работе исследована форма полосы люминесценции с максимумом $1.236 \mathrm{eV}$ и определены параметры электрон-фононного взаимодействия с использованием однокоординатной модели. Фактор Хуанга-Риса в этом случае равен 2 , а величина тепловыделения ФранкаКондона $0.04 \mathrm{eV}$. Энергия чисто электронного перехода приблизительно равна $1.28 \mathrm{eV}$. В дальнейшем эти параметры можно использовать для вычисления вероятностей электронных излучательных и безызлучательных переходов с участием центра $\mathrm{Ti}^{+3}-$ вакансия кислорода и анализа результатов фотокатализа и характеристик солнечных элементов на основе тонкопленочного диоксида титана.

\section{Финансирование работы}

Работа выполнена при поддержке Минобрнауки России, проект № 0004-2019-0002.

\section{Конфликт интересов}

Авторы заявляют, что у них нет конфликта интересов.

\section{Список литературы}

[1] Tang H., Prasad K., Sanjinès R., Schmid P.E., Lévy F. // J. Appl. Phys. 1994. V. 75. N 4. P. 2042-2047. doi 10.1063/1.356306

[2] Chao S., Wang W.H., Lee C.C. // Appl. Opt. 2001. V. 40. N 13. P. 2177-2182. doi 10.1364/AO.40.002177

[3] Yokogawa T., Yoshii S., Tsujimura A., Sasai Y., Merz J. // Jpn. J. Appl. Phys. 1995. V. 34. Part 2. N 6B. P. L751-L753. doi 10.1143/jjap.34.1751

[4] Fukuda H., Namioka S., Miura M., Ishikawa Y., Yoshino M., Nomura S. // Jpn. J. Appl. Phys. 1999. V. 38. Part 1. N 10. P. 6034-6038. doi 10.1143/JJAP.38.6034

[5] Campbell S.A., Gilmer D.C., Wang X.C., Hsieh M.T., Kim H.S., Gladfelter W.L., Yan J.H. // IEEE Trans. Electron Devices. 1997. V. 44. N 1. P. 104-109. doi 10.1109/16.554800

[6] Fujishima A., Honda K. // Nature. 1972. V. 238. P. 37-38. doi $10.1038 / 238037 \mathrm{a} 0$

[7] Mills A., Davies R.H., Worsley D. // Chem. Soc. Rev. 1993. V. 22. N 6. P. 417-25. doi 10.1039/CS9932200417

[8] Maness P.C., Smolinski S., Blake D.M., Huang Z., Wolfrum E.J., Jacoby W.A. // Appl. Environ. Microbiol. 1999. V. 65. N 9. Р. 4094-4098. [Электронный ресурс] Режим доступа: https://aem.asm.org/content/aem/65/9/4094.full.pdf

[9] Paz Y., Luo Z., Rabenberg L., Heller A. // J. Mater. Res. 1995. V. 10. N 11. P. 2842-2848. doi 10.1557/JMR.1995.2842

[10] Poulios I., Spathis P., Grigoriadou A., Delidou K., Tsoumparis P. // J. Environ. Sci. Health Part A. 1999. V. 34. N 7. P. 1455-1471. doi 10.1080/10934529909376905

[11] O'Regan B., Gratzel M. // Nature. 1991. V. 353 P. 737-740. doi 10.1038/353737a0

[12] Xи Y., Yao K., Zhou X., Cao Q. // Sens. Actuators B: Chem. 1993. V. 14. N 1-3. P. 492-494. doi 10.1016/09254005(93)85061-E

[13] Phillips L.G., Barbano D.M. // J. Dairy Sci. 1997. V. 80. N 11. P. 2726-2731. doi 10.3168/jds.S0022-0302(97)76234-9

[14] Tryk D.A., Fujishima A., Honda K. // Electrochimica Acta. 2000. V. 45. N 15-16. P. 2363-2376. doi 10.1016/S00134686(00)00337-6

[15] Zhang D., Ma X., Zhang H., Liao Y., Xiang Q. // Materials Today Energy. 2018. V. 10. P. 132-140. doi 10.1016/j.mtener.2018.08.018

[16] Булярский С.В., Грушко Н.С., Жуков А.В. // ЖЭТФ. 1999. Т. 116. № 3 (9). С. 1027-1034. [Электронный pecypc]. Режим доступа: http://www.jetp.ac.ru/cgibin/dn/r_116_1027.pdf; Bulyarskii S.V., Grushko N.S., Zhukov A.V. // JETP. 1999. V. 89. N 3. P. 547-551. doi $10.1134 / 1.559013$

[17] Булярский С.В., Грушко Н.С., Жуков А.В. // Опт. и спектр. 2000. T. 88. № 3. C. 415-418; Bulyarskii S.V., Grushko N.S., Zhukov A.V. // Optics and Spectrosc. 2000. V. 88. N 3. P. 368371. doi $10.1134 / 1.626822$ 
[18] Swamy V. // Phys. Rev. B. 2008. V. 77. N 19. P. 195414-4. doi 10.1103/PhysRevB.77.195414

[19] Lax M. // J. Chem. Phys. 1952. V. 20. N 11. P. 1752-1760. doi 10.1063/1.1700283

[20] Ребане К.К., Пурга А.П., Сильд О.И., Хижняков В.В. // Труды ИФА АН ЭССР. 1961. Т. 14. С. 31-47.

[21] Ребане К.К., Пурга А.П., Сильд О.И., Хижняков В.В. // Труды ИФА АН ЭССР. 1961. Т. 14. С. 48-75.

[22] Булярский С.В., Радауцан С.И. // Докл. АН СССР. 1976. T. 231. № 3. С. 372-376.

[23] Булярский С.В. // Письма в ЖТФ. 1976. Т. 2. № 4. С. 102 105.

[24] Tang H., Berger H., Schmid P.E., Lévy F., Burri G. // Sol. Status Commun. 1993. V. 87. N 9. P. 847-850. doi 10.1016/0038-1098(93)90427-O

[25] Гнеденко Б.В. Курс теории вероятностей. М.: Наука, 1988. 448 c.; Gnedenko B.V. Theory of Probability. Mir Publishers, 1988. $392 \mathrm{p}$.

[26] Powell M.J.D. // Computer J. 1964. V. 7. N 2. P. 155-162. doi 10.1093/comjn1/7.2.155

[27] Химмельблау Д. Прикладное нелинейное программирование. М.: Мир, 1975. 534 c.; Himmelblau D.M. Applied Nonlinear Programming. McGraw-Hill, 1972. 498 p. 